Original Article, Cardiology

\title{
Effect of Subclinical Thyroid Disease on Cardiac Function in Patients on Thyroid Replacement Therapy as Assessed by Radionuclide Ventriculography
}

\author{
Elsayed, $\mathbf{Y}^{\mathbf{1}}$. Hasanin, $\mathbf{E}^{\mathbf{2}}$.Farouk, $\mathrm{Sh}^{\mathbf{3}}$. Zeiada, $\mathbf{G}^{4}$. \\ ${ }^{1}$ Nuclear Medicine Unit, Faculty of Medicine, Cairo University. ${ }^{2}$ Nuclear Medicine Unit, \\ South Egypt Cancer Institute, Assuit University, ${ }^{3}$ Nuclear Medicine Unit, Faculty of \\ Medicine, Zagazig University, Egypt. ${ }^{4}$ Nuclear Medicine Unit, Faculty of Science, Kuwait \\ University.
}

\begin{abstract}
:
Objective: The evidence indicates that the cardiovascular system responds to the minimal but persistent changes in circulating thyroid hormone levels, which is typical of individuals with subclinical thyroid dysfunction. However, there is no study to compare between control or euthyroid and subclinical thyroid cases with tong-term L-thyroxine therapy regarding cardiac function of the heart using radionuclide ventriculography. The objective of our study is to assess and compare the left ventricular function through the evaluation of the radionuclide systolic parameters, diastolic parameters and heart rate for these conditions. Methods: Ten healthy controls and 32 patients with surgically treated differentiated thyroid carcinoma followed by radioactive iodine ablation and receiving suppressive L-thyroxine were evaluated by gated radionuclide

divided into 3 groups according to their thyroid hormone profile. These groups were: euthyroid, subclinical hypothyroid, subclinical hyperthyroid groups. Results: Long-term L-thyroxine therapy significantly affected the left ventricular systolic function in patients with subclinical thyroid dysfunction. It prolonged TPER and TPFR in patients with subclinical hypothyroid dysfunction while it decreased TPER and TPFR in patients with subclinical hyperthyroid dysfunction. However, there was no effect on the euthyroid group. Conclusions: These abnormalities in cardiac function in subclinical hypo- or hyperthyroidism may lead to long-term effect on patient's lives. Early effective and accurate treatment for these patients is important to avoid the consequences of long term exposure of the cardiovascular system to small increases or decrease of thyroid hormone.
\end{abstract} ventriculography. The patients were

Key Words: Heart, subclinical hypo or hyperthyroidism, thyroid hormones. 


\section{INTRODUCTION:}

Subclinical hyper- or hypothyroidism is asymptomatic conditions with apparently normal serum free thyroxine (T4) and free triiodothyronine (T3) levels. Subclinical thyroid diseases diagnoses are based on laboratory evaluation. Subclinical hypothyroidism, defined by elevated serum levels of thyroid stimulating hormone (TSH) with normal serum levels of free thyroid hormones, ${ }^{(1)}$.Among individuals with this condition, up to half of them may progress to overt thyroid failure. When lasting more than 6-12 months, it may be associated with abnormal lipid metabolism, a subtle cardiac defect with mainly diastolic dysfunction, impaired vascular function, elevated risk of atherosclerosis and ischemic heart disease (2). Restoration of euthyroidism by LT4 treatment may correct the cardiac abnormalities $(3,4)$. Subclinical hyperthyroidism is an increasingly recognized entity that is defined as a normal serum free thyroid hormones levels with suppressed TSH below the normal range (2). It has been reported that subclinical hyperthyroidism is not associated with coronary heart disease but induces arrhythmias including arterial fibrillation and arterial flutter ${ }^{(2)}$. The more frequent causes of endogenous subclinical hyperthyroidism are toxic adenoma and Graves' disease; whereas the exogenous causes are induced by T4 therapy used to suppress TSH in patients with nontoxic goiter and differentiated thyroid cancer (DTC) ${ }^{(5)}$.The evidence indicates that the cardiovascular system responds to the minimal but persistent changes in circulating thyroid hormone levels, which is typical of individuals with subclinical thyroid dysfunction $^{(3)}$. Although the effects of thyroid hormones, overt hyper-and hypothyroidism on the cardiovascular system have been diffusely studied (7-12) Only in the last years the effects of acute subclinical hyper- and hypothyroidism on the heart have been investigated (2, 6). Therefore subclinical thyroid dysfunction is currently the subject of many studies and remains controversial, particularly as it relates to cardiovascular effects and clinical applications. This study evaluates and compares the effects of subclinical thyroid disease on cardiac function in post I-131 ablation thyroid cancer patients on thyroid replacement therapy as assessed by radionuclide ventriculography.

\section{PATIENTS AND METHODS:}

The study population included 32 patients, male $\&$ females (mean age, $45 \pm 6.8$ year) of differentiated thyroid carcinoma. All patients were receiving thyroid hormone replacement therapy (L-thyroxine) at the time of their enrolment in this study. In all patients, as per the routine practice, the treatment with L-thyroxine started with a dose $25 \mu \mathrm{g}$, and then increased gradually according to the medical need. The study population also consisted of ten healthy control, age matched (group 1). The patients were classified into 3 groups according to the level of T3, T4 and TSH as determined using radioimmunoassay with IMMUNOTECH kits. These groups were as follows: group 2 euthyroid cases (7 patients), group 3 subclinical hypothyroid cases (14 patients); group 4 subclinical hyperthyroid cases (18 patients). All patients entered the study only if they had shown stable subclinical hypo or hyper thyroidism according to their group for at least 1 year before the study with TSH level $>5 \mathrm{~m} \mathrm{U} / \mathrm{L}$ and $<0.2 \mathrm{~m} \mathrm{U} / \mathrm{L}$ respectively. 
Cardiovascular and respiratory diseases or other systemic diseases were excluded in both patients and control subjects by a complete clinical work-up. Routine laboratory chemistry was normal in all, and none of the patients was being treated for any other clinical condition except for their primary cancer disease and only (4) patients were on beta blockers, The study protocol was reviewed approved by the institutional ethics committee; and all patients gave their informed written consent to this study. Radioimmunoassay: Serum T4, T3 and TSH were determined by a homogenous radio-immunoassay (IMMUNTECH kits). TSH is measured by radioimmunoassay in a third generation gamma counter.

Gated Radionuclide Ventriculography: Standard protocol for in vivo labeling of RBCs with Tc-99m was followed. Stannous pyrophosphate $(10 \mu \mathrm{g} / \mathrm{Kg})$ was injected intravenous to the patient. Tc-99m pertechnetate (20-25 mCi) was injected 1530 minutes later. The patient was put in supine position under a gamma camera $(A D A C)$ connected to ECG monitor and a computer. The camera head was adjusted in the left anterior oblique (LAO) view at $45^{\circ}$ to the longitudinal axis of the body for the best septal separation of both ventricles. Gated acquisition of data was carried out and processed by analysis an area of interest (MX) around the left ventricle to get: Left ventricular end diastolic counts (LVEDC), left ventricular end systolic counts (LVESC), left ventricular ejection fraction (LVEF), differentiation of the left ventricular volume curve to obtain: Time to peak ejection rate (TPER), time to peak filling rate (TPFR), peak ejection rate (PER) \& peak filling rate (PFR).

Statistical analysis: Data were presented as mean \pm S.E.M of (n) experiments. Where necessary, differences between two mean values were compared using Student's test paired or unpaired as appropriate. Where multiple comparisons were necessary, oneway analysis of variance (ANOVA) was used followed by Post Hoc Test (Benferroni). The difference was assumed to be significant at $\mathrm{P}<0.05$.

\section{RESULTS:}

Thyroid hormones: A total of 32 patients were recruited for this study. The general characteristics of the study patients are summarized in (Table 1). The duration of thyroxine therapy was 3-14 yr. The mean L-thyroxine dosage was 200-300 $\mu \mathrm{g} /$ day (220 $\pm 22 /$ day). Mean serum T3 and T4 were increased in groups 4 (subclinical hyperthyroidism); while they decreased in groups 3 (subclinical hypothyroidism). The mean serum TSH concentration determined on the day of evaluation confirmed the chart review that TSH was suppressed to near the limits of assay detection $(<0.01$ $\mu \mathrm{U} / \mathrm{mL}$ ) for group 4 and significantly increase to $46.6 \pm 18 \mu \mathrm{U} / \mathrm{mL}$ for group 3 . Mean serum T3, T4 \& TSH levels in group 2 (euthyroidism); were similar to the control, therefore patients had minimal symptoms than the control (Table 2). 
Table 1. Clinical characteristics of the study patients

\begin{tabular}{|c|c|c|}
\hline & Patients & Controls \\
\hline Number & 32 & 10 \\
\hline Age (Years) & $45 \pm 6.8$ & $47 \pm 4.9$ \\
\hline L-T4 dose & $200-300$ & - \\
\hline Duration of & $3-14$ years & - \\
\hline
\end{tabular}

Table 2. Hormonal patterns in patients during L-T4 suppressive therapy and in the control group

\begin{tabular}{|c|c|c|c|c|}
\hline Groups & Classification & $\begin{array}{c}\text { Free T3 } \\
(\boldsymbol{\mu g} / \mathbf{d L})\end{array}$ & $\begin{array}{c}\text { Free T4 } \\
(\boldsymbol{\mu g} / \mathbf{d L})\end{array}$ & $\begin{array}{c}\text { TSH } \\
(\boldsymbol{\mu U} / \boldsymbol{m L})\end{array}$ \\
\hline Group 1 & Control group & $3 \mu \mathrm{g} / \mathrm{dL}$ & 6 & $1.75 \pm 1.39$ \\
\hline Group 2 & Euthyroid & $3.81 \pm 1.26$ & $15.5 \pm 2.99$ & $1.31 \pm 1.58$ \\
\hline Group 3 & $\begin{array}{c}\text { Subclinical } \\
\text { hypothyroid }\end{array}$ & $333 \pm 126$ & $11.9 \pm 4.23 *$ & $46.6 \pm 18.8 *$ \\
\hline Group 4 & $\begin{array}{c}\text { Subclinical } \\
\text { hyperthyroidism }\end{array}$ & $4.29 \pm 0.68$ & $17.9 \pm 2.69 *$ & $0.01 \pm 0.003 *$ \\
\hline
\end{tabular}

* Significance between control and other groups $<0.05$

\section{Hemodynamic changes}

There is no significant difference between the control and all the groups for heart rate (HR). Values of HR were detected for all groups with a tendency towards elevation in group 4 (Table 3). Although patients of group 4 are controlled by B- adrenal receptor antagonist.

Table 3. Comparison of mean values of heart rate (HR)

\begin{tabular}{|c|c|c|}
\hline Groups & Classification & HR (bpm) \\
\hline Group 1 & Control group & $75.4 \pm 5.19$ \\
\hline Group 2 & Euthyroid & $74.3 \pm 4.23$ \\
\hline Group 3 & Subclinical hypothyroid & $76.6 \pm 13.8$ \\
\hline Group 4 & Subclinical hyperthyroidism & $81.3 \pm 9.93$ \\
\hline
\end{tabular}

No significance between control and other groups $>0.05$

Gated radionuclide ventriculography

\section{Systolic Function}

Systolic function parameters EF, PER \& TPER were calculated (Table 4). There were no differences between the control and the euthyroid groups. However there were significant differences between these two groups, and groups 3 (subclinical hypothyroidism) and group 4 (subclinical hyperthyroidism) in TPER. In addition, there is also a difference between group 3 \& group 4 in TPER. 
Table 4. Comparison of the mean \pm SD values for systolic function parameters

\begin{tabular}{|c|c|c|c|c|}
\hline Groups & Classification & EF (\%) & $\begin{array}{c}\text { PER } \\
\text { (EDV/s) }\end{array}$ & $\begin{array}{c}\text { TPER } \\
\text { (Sec) }\end{array}$ \\
\hline Group 1 & Control group & $63.4 \pm 7.53$ & $3.25 \pm 0.63$ & $0.146 \pm 0.0006$ \\
\hline Group 2 & Euthyroid & $57.6 \pm 5.87$ & $3.43 \pm 0.38$ & $0.147 \pm 0.0004$ \\
\hline Group 3 & Subclinical hypothyroid & $54.9 \pm 11.3$ & $3.06 \pm 0.65$ & $0.161 \pm 0.001^{*+}$ \\
\hline Group 4 & $\begin{array}{c}\text { Subclinical } \\
\text { hyperthyroidism }\end{array}$ & $59.0 \pm 7.43$ & $3.02 \pm 0.56$ & $0.144 \pm 0.003^{*+}$ \\
\hline
\end{tabular}

$E F=$ Ejection Fraction; PER =Peak Ejection Rate; TPER = Time to Peak Ejection Rate

* Significance between control and groups $3,4(p<0.05)$

\section{Diastolic Function}

Evaluation of PFR \& TPFR parameters of diastolic function was done (Table 5). There were significant differences in TPFR between the control and groups $3 \&$
4. The mean values of TPFR and PFR were compared within the groups showing differences between the group 3 and group 4.

Table 5. Comparison of the mean \pm SD values for diastolic function parameters

\begin{tabular}{|c|c|c|c|}
\hline Groups & Classification & $\begin{array}{c}\text { PFR } \\
(\text { EDV/s) }\end{array}$ & $\begin{array}{c}\text { TPFR } \\
\text { (sec) }\end{array}$ \\
\hline Group 1 & Control group & $3.24 \pm 0.68$ & $0.148 \pm 0.0006$ \\
\hline Group 2 & Euthyroid & $2.89 \pm 0.30$ & $0.153 \pm 0.0004^{+}$ \\
\hline Group 3 & Subclinical hypothyroid & $2.74 \pm 0.53^{+}$ & $0.163 \pm 0.001^{*+}$ \\
\hline Group 4 & Subclinical hyperthyroidism & $3.82 \pm 0.96^{+}$ & $0.127 \pm 0.002^{*+}$ \\
\hline
\end{tabular}

PFR = Peak Filling Rate; TPFR = Time to Peak Filling Rate * Significance between control and other groups $(P<0.05)+P<0.05$ between groups $3 \& 4$ for $P E R+P<0.05$ between groups 2,3 \& 4 fro $T P F R$

\section{DISCUSSION:}

The term subclinical thyroid disease is used to describe asymptomatic thyroid abnormalities found on imaging study or laboratory tests ${ }^{(\mathbf{1 3})}$. Subclinical hypo- or hyper-thyroidism have been associated with cardiac dysfunction ${ }^{(6)}$. Almost half of them may progress to overt thyroid failure (1). Previous studies have suggested that subclinical thyroid dysfunction, as manifested by abnormalities in TSH levels, are associated with detrimental effects on the cardiovascular system. Subclinical hypothyroidism is characterized by abnormal lipid metabolism, cardiac dysfunction, diastolic hypertension conferring an elevated risk of atherosclerosis, and ischemic heart disease. Similarly, patients with subclinical hyperthyroidism have 3 times the likelihood of arterial fibrillation ${ }^{(14)}$. Our study showed that subclinical hypo- \& hyper-thyroid groups had abnormality in diastolic and systolic parameters. It had higher or lower TPFR and TPER than the 
control. There are few studies in the past have reported abnormal left ventricular systolic function ${ }^{(15-17)}$ which were proven by the present study. Whereas impaired myocardial contractility in overt hypothyroidism has been documented clinically and experimentally, the presence of similar alterations in subclinical hypothyroidism is still under debate. An impairment of left ventricular systolic function has been reported in subclinical hypothyroid patients both at rest and during exercise, with a clear improvement after L$\mathrm{T} 4$ replacement therapy ${ }^{(18)}$. It was also reported an impairment of left ventricular diastolic function was reversed by 6 months of L-T4 replacement therapy ${ }^{(1)}$. However other researchers did not find alterations of systolic time intervals or ejection fraction, as assessed by simultaneous recording of aortic and mitral flow velocities. It was reported that there is impairment of both left ventricular diastolic and systolic function in subclinical hypothyroidism $^{(19)}$. Subclinical hypothyroidism is associated with impaired left ventricular diastolic function at rest, systolic dysfunction on effort, and enhanced risk for atherosclerosis and myocardial infarction. Subclinical hyperthyroidism is associated with increased heart rate, arterial arrhythmias, increased left ventricular mass with marginal concentric remodeling, impaired ventricular relaxation, reduced exercise performance, and increased risk for cardiovascular death. All abnormalities were reversed by restoration of euthyroidism or were blunted by Bblockade and tailoring of the L-thyroxine dose. In this study there is no significant difference of HR between the control and all the groups. This is mainly because the HR of group 4 is treated by B-adrenal receptor antagonist. It regulates the heart rate and prevents the tachycardia. The significant increase in heart rate has many implications in patients. Enhanced heart rate has been shown to be associated with increased risk of cardiovascular and noncardiovascular mortality ${ }^{(20)}$. Heart rate is an important mechanism for regulation of cardiac output. Apart from determining the rate of cardiac ejection, HR can affect both systolic and diastolic function ${ }^{(5)}$.In our study, euthyroid group showed no difference than the control group in all the investigated parameters, starting with the level of the thyroid related hormones (T3, $\mathrm{T} 4 \& \mathrm{TSH})$ and the cardiac systolic (EF, PER \& TPER) and diastolic (PFR \& TPFR) or HR which was expected. These results verify that good and accurate treatment can prevent any abnormality in the heart. The long-term replacement thyroxin therapy in patients with differentiated thyroid carcinoma who underwent surgery followed by radioiodine ablation may significantly affects the left ventricular function. It impairs the diastolic and systolic function in patients with subclinical hyper- and hypo-thyroidism. It decreased TPER and TPFR in subclinical hyper-thyroid patients and prolonged them in hypothyroid patients. It was known that patients chronically treated with suppressive doses of L-T4 were usually free of symptoms and signs of thyrotoxicosis. However our results showed that they could suffer from left ventricular abnormality function if there is no continuous care. Therefore, early effective treatment of subclinical thyroid diseases is important for differentiated thyroid carcinoma patients to improve their quality of life and to avoid the consequences of long term exposure of the cardiovascular system to small increases or 
decreases of thyroid hormone. Therefore we recommend deciding whether thyroxine therapy has a long-term benefit that outweighs the risks of cardiovascular before starting the treatment in each individual separately.

\section{REFERENCES:}

1. Palmieri EA, Fazio S, Lombardi G, Biondi B. Subclinical hypothyroidism and cardiovascular risik: a reason to treat?. Treat Endocrinol.; 3(4): 233-244, 2004.

2. Dattilo G, Crosca S, Tavella S, Marte F, Patttane S. Pericardial effusion associated with subclinical hypothyroidism. Int $\mathrm{J}$ Cardiol.; ahead of print, 2009.

3. Vanderpump MP, Tunbridge WM, French JM, Appleton D, Bates D, Clark F, Grimley Evans J, Hasan DM, Rodgers

$\mathbf{H}$, Tunbridge F, et al. The incidence of thyroid disorders in the community: a twenty-year follow-up of the Whickham Survey. Clin Endocrinol.;43(1):55-68, 1995.

4. Vanderpump MP, Tunbridge WM. The effects of drugs on endocrine function. Clin Endocrinol (Oxf);;39 (4): 389-97, 1993.

5. Biondi B, Fazio 5, Sacca L, et al. Effects of chronic subclinical hyperthyroidism from levothyroxine on cardiac morphology and function. Cardiologia.; 44 (5): 443-9, 1999.

6. Rodondi N, Bauer DC, Cappola AR, Cornuz J, Robbins J, Fried LP, Ladenson PW, Vittinghoff E, Gottdiener JS, Newman AB. Subclinical thyroid dysfunction, cardiac function, and the risk of heart failure. The cardiovascular health study. J Am Coll Cardiol; 52(14):1152-1159, 2008.

7. Akincioglu C, Berman DS, Nishina H, Kavanagh P, Slomka P, Abidov A, Hayes S, Friedman JD, Germano G. Assessment of diastolic function using 16 -frame $99 \mathrm{mTc}$ sestamibi gated myocardial perfusion
We conclude that careful clinical evaluation, continuous cardiac laboratory studies, is sufficient to manage the patients with long-term thyroxine therapy.

SPECT: normal values. J Nucl Med.; 46 (7):1102-1108, 2005.

8. Fazio S, Palmieri EA, Lombardi G, Biondi B. Effects of thyroid hormone on the cardiovascular system. Recent Prog Horm Res.; 59:31-50, 2004.

9. Biondi B, Palmieri EA, Lombardi G, Fasio S. Effects of thyroid hormone on cardiac function: the relative importance of heart rate, loading conditions and myocardial contractility in the regulation of cardiac performance in human hyperthyroidism. J Clin Endocrinol Metab; 87(3):968-974, 2009.

10. Cappola AR, Fried LP, Arnold AM, Danese MD, Kuller LH, Burke GL, Tracy RP, Ladenson PW. Thyroid status, cardiovascular risk, and mortality in older adults. JAMA.; 295 (9): 1033-1041, 2006.

11. Tielens ET, Pillay M, Storm C, Berghout A. Cardiac function at rest in hypothyroidism evaluated by equilibrium radionuclide angiography. Clin Endocrinol (Oxf).; 50 (4): 497-502, 1999.

12. Klein I, Ojamaa K. Thyrotoxicosis and the heart. Endocrinol Metab Clin North Am.; 27 (1): 51-62, 1998

13. Fatourechi V.Adverse effects of subclinical hyperthyroidism.Lancet Oncology Journal ; 15; 856-7, 2001.

14. Duggal J, Singh S, Barsano CP, Arora R. Cardiovascular risk with subclinical hyperthyroidism and hypothyroidism: pathophysiology and management.;2 (3): 198-206, 2007. 
15. Biondi B, Fazio S, Carella C, Amato G, Cittadini A, Lupoli G, et al. Cardiac effects of long term thyrotropin-suppressive therapy levothyroxine. J Clin Endocrinol Metab.; 77 (2):332-3, 1993.

16. Khanna CM, Dubey YS, Shankar R, Kaur G. Effects of long-term thyroid hormone suppressive treatment on the cardiac functions. Indian Heart J.; 49(3):28992, 1997.

17. Boutin JM, Matte R, D'Amour P, Gilbert F, Havrankova J, Belanger R, Chartrand R, Zakarija M. Characteristics of patients with normal T3 and T4 and a low TSH response to TRH. Clin Endocrinol. 1986;25(5):579-88. 1986.

18. Vanderpump MP, Tunbridge WM. The effects of drugs on endocrine function. Clin Endocrinol :Oct;39(4):389-97, 1993.
19. Biondi B, Palmieri EA, Lombardi G, Fazio S. Effect of thyroid hormone on cardiac function: the relative importance of heart rate, loading conditions and myocardial contractility in the regulation of cardiac performance in human hyperthyroidism. $\mathrm{J}$ Clin Endocrinol Metab.; 87(3):968-974, 2002.

20. Monzani F, Di Bello V, Caraccio N, Bertini A, Giorgi D, Giusti C, Ferrannini E. Effect of levothyroxine on cardiac function and structure in subclinical hypothyroidism: a double blind, placebocontrolled study. J Clin Endocrinol Metab.;86(3):1110-5,2001. 